\title{
Cosmic ray astroparticle physics: current status and future perspectives
}

\author{
Fiorenza Donato \\ Department of Physics, Torino University, and INFN, Sezione di Torino \\ via P. Giuria 1, 10125 Torino, Italy \\ email: donato@to.infn.it
}

\begin{abstract}
The data we are receiving from galactic cosmic rays are reaching an unprecedented precision, over very wide energy ranges. Nevertheless, many problems are still open, while new ones seem to appear when data happen to be redundant. We will discuss some paths to possible progress in the theoretical modeling and experimental exploration of the galactic cosmic radiation.
\end{abstract}

Keywords. Cosmic rays, dark matter, Galaxy.

\section{Introduction}

A large contribution to the knowledge and understanding of the Galaxy is given by the observation of the most energetic particles, the CRs (CRs). These relativistic particles, reaching the Earth from the outer space, are either primary nuclei, arriving directly from their sources, or secondary products of the spallation processes (i.e. fragmentation by nuclear destruction) taking place during the propagation from the sources through the interstellar medium (ISM). They include nuclei from hydrogen to very high Z, and possibly in different isotopic compositions, electrons, antimatter (positrons and antiprotons measured up to now), and in some extensions also gamma rays.

The main challenges we are facing are i) the identification of the sources of CRs, ii) the mechanism through which low energy particles, the seeds being single elements, dust or grains at rest in the ISM are accelerated to such high energy to be called CRs, and iii) the understanding of their propagation through the galactic magnetic fields. Due to the wide energy range explored by detectors and the rapidly changing flux, different experimental techniques are needed in diverse energy regions. Direct measurements of the primary CRs can be performed up to $10^{14} \mathrm{eV}$. Above this indicative threshold, the low fluxes due to the steeply falling spectrum force us to exploit indirect methods, detecting for example the extensive air showers generated by the interaction of CRs in the atmosphere.

Some components of the CRs are also a peculiar means for the exploration of dark matter (DM) signals coming from the Galaxy. The most common hypothesis for particle DM consists of weakly interacting massive particles (WIMPs), supposed to be cold thermal relics after the Big Bang and to build up the galactic dark haloes. The so-called indirect DM investigation technique relies in the search for its stable annihilation products in the halo of galaxies, and in particular in the Milky Way. The indirect DM detection focuses on rare components in charged CRs (CRs), as well as in $\gamma$-rays and, more generally, in multiwavelength photons (Salati, Donato \& Fornengo (2010)). 


\section{Galactic CRs}

Galactic CRs (GCR) are supposed to be produced by nucleosynthesis processes in the stellar interior, where they are fractionated, ejected and then accelerated to CR energies and diffused throughout the whole Galaxy. They reach the Solar System, where the slowest CRs experience a spectral distortion by the solar wind called solar modulation. Charged particles are bent in the galactic magnetic fields, so there is no obvious way of tracking them back to their sources. The energy spectrum which is observed at Earth is folded with the source one through an energy dependent diffusion coefficient which shapes the effects of the galactic magnetic field in an effective way, and which can be deduced only phenomenologically. These peculiarities are at variance with $\gamma$ rays, which point directly to the sources.

The most realistic propagation models are the diffusion ones, which account for spatial dependence of sources, CR densities and in principle of the ISM. The Galaxy is usually shaped as a thin gaseous disk where all the astrophysical sources are located, embedded in a thick diffusive magnetic halo. Diffusive models, besides being more realistic and closer to a physical interpretation for each component, have proven to be successful in reproducing the nuclear, antiproton and radioactive isotopes data. They also allow to treat contributions from dark matter (or other exotic) sources located in the diffusive halo.

The relevant transport equation for a charged particle wandering through the magnetic inhomogeneities of the galactic magnetic field writes in terms of the differential density $N(E, \vec{r})$ as a function of the total energy $E$ and the position $\vec{r}$ in the Galaxy. Assuming steady-state $(\partial N / \partial t=0)$, the transport equation for a given nucleus (the subscript $j$ is omitted) can be written in a compact form as

$$
\left(-\vec{\nabla} \cdot(K \vec{\nabla})+\vec{\nabla} \cdot \vec{V}_{C}+\Gamma_{\text {rad }}+\Gamma_{\text {inel }}\right) N+\frac{\partial}{\partial E}\left(b N-c \frac{\partial N}{\partial E}\right)=\mathcal{S} .
$$

The first bracket in the l.h.s. accounts for: i) spatial diffusion $K(\vec{r}, E)$, ii) convection with speed $\vec{V}_{C}(\vec{r})$, iii) the (possible, for some isotopes with half lifetime $\tau_{0}$ ) radioactive decay rate $\Gamma_{\text {rad }}(E)=1 /\left(\gamma \tau_{0}\right)$ ( $\gamma$ here is the Lorentz factor $)$, iv) the destruction rate $\Gamma_{\text {inel }}(\vec{r}, E)=\sum_{I S M} n_{\mathrm{ISM}}(\vec{r}) v \sigma_{\text {inel }}(E)$ due to collisions with the interstellar medium. In this last expression $n_{\mathrm{ISM}}(\vec{r})$ is the density of the interstellar medium in the various locations of the Galaxy and in its different $\mathrm{H}$ and $\mathrm{He}$ components, and $\sigma_{\text {inel }}(E)$ is the destruction (inelastic) cross section for a given nucleus. The coefficients $b$ and $c$ are respectively first and second order gains/losses in energy. The source term $\mathcal{S}$ includes primary sources of CRs (e.g. supernovae), secondary sources due to the fragmentation of heavier nuclei, and secondary decay-induced sources. The quasi-linear theory leads to a rigidity power law for the diffusion coefficient, which is usually assumed to have the form:

$$
K(E)=K_{0} \beta^{\eta} R^{\delta}
$$

( $\beta$ is the Lorentz factor). $K_{0}$ is linked to the level of the hydromagnetic turbulence and $\delta$ to the density spectrum of these irregularities at different wavelength. $\eta$ is usually set to 1 , while a different value parameterizes very low energy deviations. The lack of information on the magnetic field irregularities prevents us from a precise determination of the diffusion coefficient, which is instead possible only from interpretation of CR data. The most important observable at these regards is the ratio $\mathrm{B} / \mathrm{C}$ of the boron to carbon fluxes. It is considered a kind of standard candle in the CR phenomenology, being the ration of an almost secondary species to an almost primary one. 
The solution to the diffusion equation (Eq.2.1) has been deeply investigated and several different techniques proposed in the literature can lead to similar fluxes at the Earth, at least for stable nuclei (Strong et al. (2007), Maurin et al. (2002))

Semi-analytical models are treated in Maurin et al. (2001). Fully numerical solution is the one adopted by the GALPROP code Strong \& Moskalenko (1998), which is particularly useful if aiming at the production of gamma rays from charged CRs. Gamma rays do not diffuse and are therefore observed at their source, so that a full spatial treatment is required. Comparable results for the propagation of stable primary and secondary nuclei have been obtained with the similar Dragon code (Evoli et al. (2008), Evoli et al. (2017)). Recently, the PICARD code has been presented as well in Kissmann (2014). For a full treatment of galactic CRs physics we refer to Castellina \& Donato (2012).

\section{Antimatter in CRs}

The presence of a small amount of antimatter in CRs is predicted from spallation reactions of incoming protons and helium nuclei on the ISM (contributions from CRs with higher $\mathrm{Z}$ being negligible). The spallation products of these inelastic scatterings account for quarks and gluons, which immediately hadronize. In particular, the hadronization process includes the production of a small amount of antiprotons, antideuterons and positrons (the latter induced by decay processes). Antiprotons and positrons in CRs have been measured in recent years with increasing accuracy and in an energy window spanning from few hundreds of $\mathrm{MeV}$ to hundreds of $\mathrm{GeV}$. For antideuterons, only upper limits have been set.

The search for cosmic antimatter is a further test of the propagation model. The study of light antimatter, due to its tiny flux, is optimal to search for contributions with spectral peculiarities, such as non-thermal production or annihilation from dark matter (DM) particles in the galactic dark halo. The latter are usually predicted to be Weakly Interacting Massive Particles (WIMPs).

The indirect dark matter detection is based on the search for anomalous components due to the annihilation of DM pairs in the galactic halo, in addition to the standard astrophysical production (Salati, Donato \& Fornengo (2010), Donato et al. (2004)). These contributions are potentially detectable as spectral distortions in various cosmic antimatter fluxes, as well as $\gamma$ rays and $\nu$ 's

$$
\chi+\chi \rightarrow q \bar{q}, W^{+} W^{-}, \ldots \rightarrow \bar{p}, \bar{D}, e^{+} \gamma \& \nu^{\prime} s .
$$

The search for exotic signals in cosmic antimatter is motivated by the very low astrophysical counterpart, acting as a background. Detection of the DM annihilation products has motivated the spectacular development of several new experimental studies.

\subsection{Antiprotons}

Antiprotons $(\bar{p})$ may be produced by spallation of high-energy primary nuclei impinging on the atoms of the ISM inside the galactic disc (Donato et al. 2001). They represent the background when searching for small peculiar contributions such as signals from DM annihilation.

The secondary antiproton flux has been predicted with small theoretical uncertainties (Donato et al. 2001) and reproduces astonishingly well the data from $200 \mathrm{MeV}$ up to at $100 \mathrm{GeV}$ (Donato et al. 2010). This can be seen in Fig. 1 where we plot, along with the solar-demodulated $\bar{p} / p$ data (data corrected by the effect of the solar wind), the curves bounding the propagation uncertainty on the $\bar{p}$ calculation. We also illustrate the uncertainty related to the production cross sections. The secondary contribution alone 


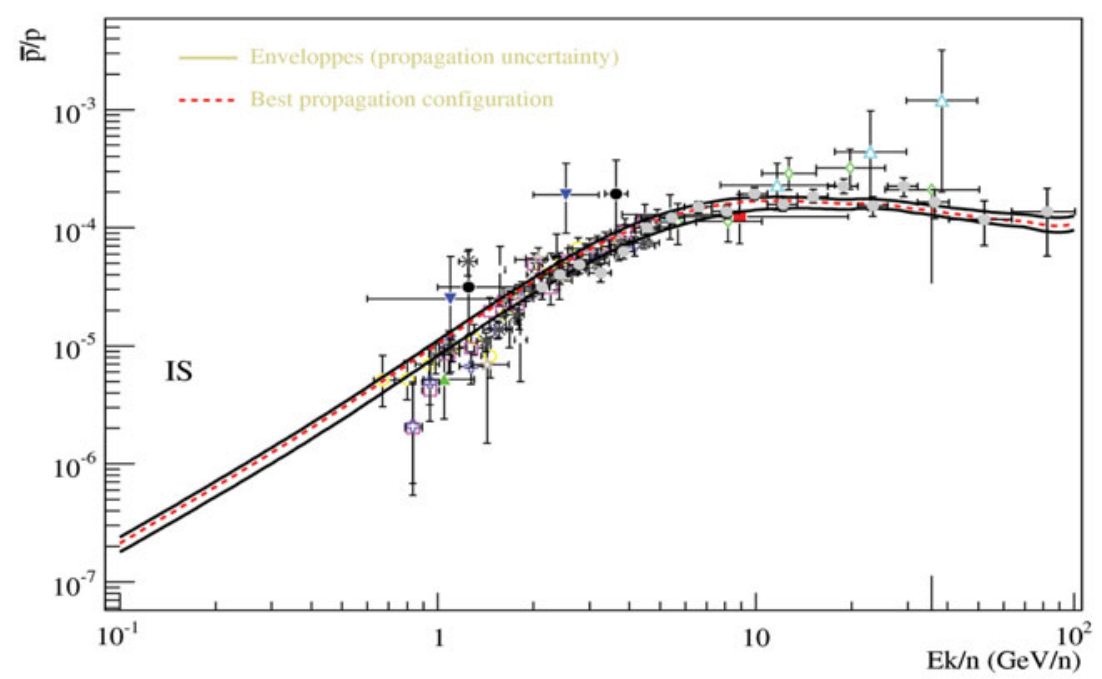

Figure 1. Propagation uncertainty envelopes of the IS $\bar{p} / p$ ratio and two parameterizations of the production cross section (see Donato et al. (2010)).

explains the PAMELA data on the whole energetic range. It is not necessary to invoke an additional component (such as, for instance, from DM annihilation in the galactic halo - see below) to the standard astrophysical one.

The annihilation of DM candidate particles throughout the whole Milky Way volume may generate primary antiprotons. In this case the WIMP annihilations take place all over the diffusive halo. The antiproton signal from annihilating DM particles leads to a primary component directly produced throughout the diffusive halo. The variation of the astrophysical parameters induces a much larger theoretical uncertainty on the primary than on the secondary flux: in the first case, the uncertainty reaches two orders of magnitude for energies $T_{\bar{p}}=1 \mathrm{GeV}$, while in the second case it never exceeds $25 \%$ (see Fig. 1 for the secondary population; for the primary DM component, see Fig. 3). The reason is in the location of the sources: the primary flux due to dark matter annihilation originates in the whole diffusive halo and is very sensitive to the halo size (which is varied between 1 and $15 \mathrm{kpc}$ ) and the convective velocity. It is of the utmost importance to constrain the propagation parameters in order to evaluate any possible exotic contribution to the $\bar{p}$ flux from sources in the whole halo.

In addition to propagation uncertainties, we notice that the secondary flux, produced by the spallation of CR protons and helium nuclei on the ISM, is affected by nuclear uncertainties. As shown in Fig. 1, they are dominated by uncertainties in the production cross sections, in particular the $p p, p H e, H e p, H e H e$ inclusive cross sections into $\bar{p}+\mathrm{X}$. We have very recently determined the characteristics that a high energy experiment should have in order to measure the $p p$ cross section, and similarly the other ones, with an accuracy so good not to exceed the errors on the AMS-02 antiproton data (Donato et al. 2017). In Fig. 2 we give our results as functions of the kinematical variables in both the LAB and CM reference frames. It shows the parameter space that has to be covered in order to guarantee the AMS-02 precision level on the $\bar{p}$ source term, if the $p+p \rightarrow \bar{p}+X$ cross section is determined with $3 \%$ uncertainty within the blue shaded regions and by $30 \%$ outside the contours. The plot is done for the LAB (left panel, a) and CM (right panel, b) reference frame variables. For the LAB frame we show the contours as functions of $\eta$ and $T$, for selected values of $T_{\bar{p}}$ from 1.1 (the lowest energy) below $30 \%$ 

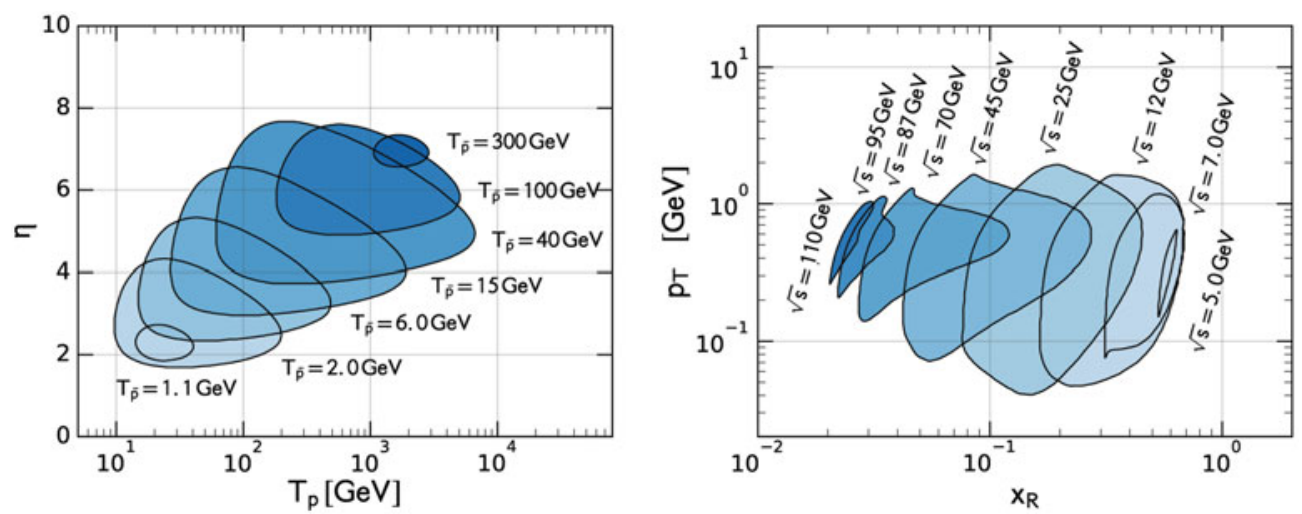

Figure 2. Parameter space of the $p p$ to $\bar{p}$ cross section necessary to determine the antiproton source term with the accuracy reached by recent AMS-02 measurements. Here we require that the cross section has to be known by $3 \%$ within the blue shaded regions and by $30 \%$ outside the contours. The left (right) panel displays the result for the LAB (CM) reference frame variables.

uncertainty in the $\mathrm{CR} \bar{p}$ flux to $300 \mathrm{GeV}$. As expected the contour size decreases when $T_{\bar{p}}$ approaches to $1 \mathrm{GeV}$, because there the AMS-02 uncertainty on the antiproton flux reaches $30 \%$. A similar explanation holds for large $T_{\bar{p}}$. Antiprotons of increasing energy require the coverage of increasing $\eta$ values. For example, the $\sigma_{\text {inv }}(p+p \rightarrow \bar{p}+X)$ at $T_{\bar{p}}=2 \mathrm{GeV}$ is known at $3 \%$ level if data were taken with proton beams between 10 and $200 \mathrm{GeV}$ and pseudorapidity from 1.8 to 4 . If the whole AMS-02 energy range had to be covered with high precision, one should collect $p+p \rightarrow \bar{p}+X$ cross section data with proton beams from $10 \mathrm{GeV}$ to $6 \mathrm{TeV}$, and $\eta$ increasing from 2 to nearly 8 . We address to Donato et al. 2017 for all the details.

\subsection{Antideuterons}

It was shown that the antideuteron spectra deriving from DM annihilation are expected to be much flatter than the secondary astrophysical component at low kinetic energies, $T_{\bar{d}}=2-3 \mathrm{GeV} / \mathrm{n}$, thus offering a potentially very clear indirect detection channel (Donato, Fornengo \& Salati (2000), Donato, Fornengo \& Maurin (2008)). Antideuterons have not been measured so far, and only upper limits exist. The dedicated GAPS experiment has been recently approved and will fly in Antarctica in few years (Aramaki et al. (2016)).

The secondary $\bar{D}$ flux is the sum of the six contributions corresponding to $p$, He and $\bar{p}$ $\mathrm{CR}$ fluxes impinging on $\mathrm{H}$ and He IS gas (other reactions are negligible). The solution to the propagation equation has the same expression as for secondary antiprotons (Donato, Fornengo \& Maurin (2008)). The production of cosmic antideuterons is based on the fusion process of a $\bar{p}$ and $\bar{n}$ pair - being the pair produced from CRs collisions or from DM annihilation in the dark galactic halo. One of the simplest but powerful treatment of the fusion of two or more nucleons is based on the so-called coalescence model which, despite its simplicity, is able to reproduce remarkably well the available data on light nuclei and antinuclei production in different kinds of collisions.

We present in Fig. 3 a possible experimental scenario. The secondary $\bar{D}$ flux is plotted alongside the primary flux from DM particle $\left(m_{\chi}=50 \mathrm{GeV}\right)$ annihilating in the halo. The three curves bound the propagation uncertainties which - as for the case of $\bar{D}$ from galactic DM, grossly rescaled by a factor $10^{3}$ - span almost two orders of magnitude on the whole energetic range. The present BESS upper limit is at a level of $2 \cdot 10^{-4}$ $\left(\mathrm{m}^{2} \mathrm{~s} \text { sr } \mathrm{GeV} / \mathrm{n}\right)^{-1}$. We also plot the estimated sensitivities of the gaseous antiparticle spectrometer GAPS on a long duration balloon flight (LDB) and an ultra-long duration 


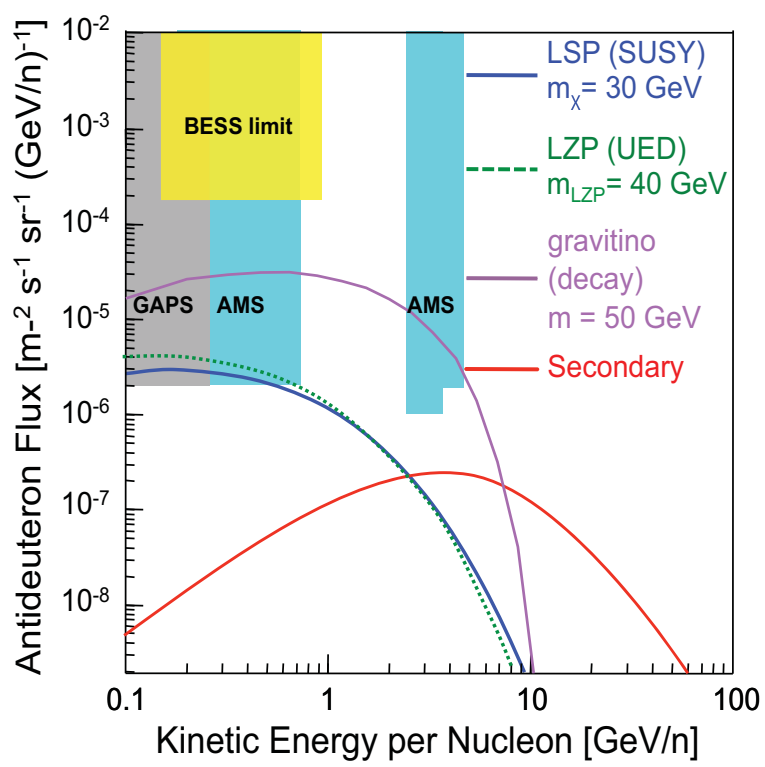

Figure 3. Predicted antideuteron flux as a function of kinetic energy per nucleon for a $30 \mathrm{GeV}$ neutralino, a $40 \mathrm{GeV}$ extra-dimensional Kaluza-Klein neutrino, and a $50 \mathrm{GeV}$ gravitino. The antideuteron limits from BESS are shown, along with the projected sensitivities of AMS-02 after 5 years of operation and GAPS after three 35-day flights. The MED Galactic propagation scenario is assumed. These predictions use a coalescence momentum that is set to $195 \mathrm{MeV}$ and the Einasto dark matter density profile. See Aramaki et al. 2016 for details.

balloon mission (ULDB) and of AMS-02 for three years of data taking. The perspectives to explore a part of the parameter space where DM annihilations are mostly expected (i.e. the low-energy tail) are promising. We refer to Aramaki et al. (2016) for details and references.

\subsection{Positrons}

Secondary positrons are produced - like antiprotons and antideuterons - by the spallation of the interstellar medium when impinging high-energy particles (Salati, Donato \& Fornengo (2010)). The main production channel is the collision of protons with hydrogen atoms at rest producing charged pions $\pi^{ \pm}$which decay into muons $\mu^{ \pm}$. The latter are also unstable and eventually lead to electrons and positrons. Positrons may also be produced through kaons although this channel is rare. In the case of positrons and electrons, Eq.2.1 describing the propagation of CRs throughout the $\mathrm{DH}$ is dominated by space diffusion and energy losses. Above a few $\mathrm{GeV}$, synchrotron radiation in the galactic magnetic fields as well as inverse Compton scattering on stellar light and on CMB photons dominate.

The positron fraction $e^{+} /\left(e^{+}+e^{-}\right)$has been measured by the PAMELA satellite experiment and confirmed by the AMS-02 exeriment, which also measures absolute leptons fluxes. It increases with energies, at variance with the predictions from pure secondary production of cosmic positrons. A viable explanation of the experimental result resides in the additional contribution of astrophysical sources accelerating leptons in their sites. The cosmic fluxes of positrons and leptons are quite sensitive to the presence of sources in the near Galaxy (few kpc), whose physics can be explored also in this peculiar channel. This result is illustrated in Fig. 6, where the $e^{+} /\left(e^{+}+e^{-}\right)$has been calculated adding to the secondary production (mostly relevant for positrons) the contributions from standard astrophysical sources, such as supernova remnants and pulsars. The most recent 


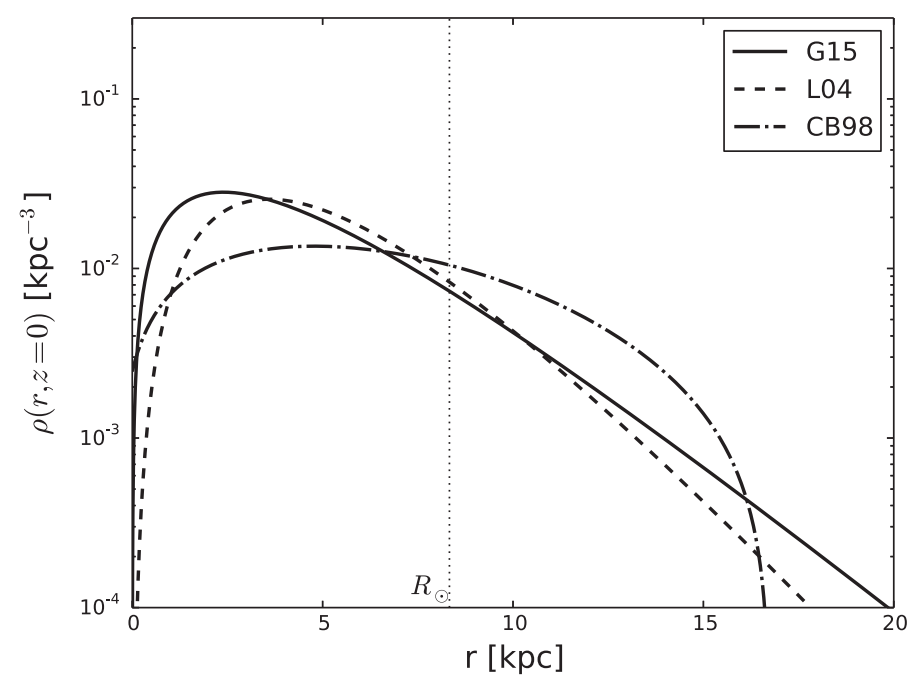

Figure 4. Radial distributions for SNRs and pulsars. The solid line corresponds to the G15 Green (2015) model. A black dotted line indicates the Earth position. See Manconi et al. (2017) for details.

experimental data on $e^{+}+e^{-}$and $e^{+}$spectra are displayed together with predictions for fluxes from galactic sources, as well as the secondary component. We find a very good fit to AMS data, with source parameters consistent with literature results. We refer to Manconi et al. (2017) for all the details and the relevant references.

\section{Anisotropies in the electrons}

Recently, the lepton data have also been analyzed in terms of their arrival direction. Indeed, no significant anisotropy has been detected, and upper limits on the dipole term $\Delta$ have been set for the electron plus positron flux. Indeed, the dipole anisotropy in the leptons arriving at the Earth can be a remarkable observable to constrain the properties of astrophysical emitters of $e^{+}$and $e^{-}$. In particular, as visible in Fig. 6, leptonic data can be interpreted with the emission of electrons and positrons from Supernova Remnants (SNRs), Pulsar Wind Nebulae (PWNe) and the secondary production given by the spallation reactions of primary CRs with the nuclei of the interstellar medium (ISM).

In Fig. 4 we reproduce the radial profile G15, together with few other largely used models. obtained from pulsars in the ATNF catalog (L04) and SNR observations (CB). The L04 and G15 models are similar around the Solar System, while they show a remarkable difference toward the Galactic center. At variance, the $\mathrm{CB} \rho(r)$ is significantly flat for most of radii. Since electrons detected at the Earth have been produced by nearby (very few $\mathrm{kpc}$ ) sources, we are mostly interested in the differences in the profiles around the Solar System. In Fig. 5 we plot our sample of near SNRs together with the most powerful PWNe projected on the Galactic plane. The source position is identified by the Galactic longitude $(l, \mathrm{deg})$ and distance $(d, \mathrm{kpc})$ to the Earth. The color scale reflects the intensity of the integrated electron flux at the Earth from $E=50 \mathrm{GeV}$ up to $E=5 \mathrm{TeV}$, which is an important observable when computing the anisotropy from a single source. The most powerful PWNe Monogem, Geminga and J2043+2740, give an integrated electron flux above $10^{8}\left(\mathrm{~cm}^{2} \mathrm{~s} \mathrm{sr}\right)^{-1}$. 


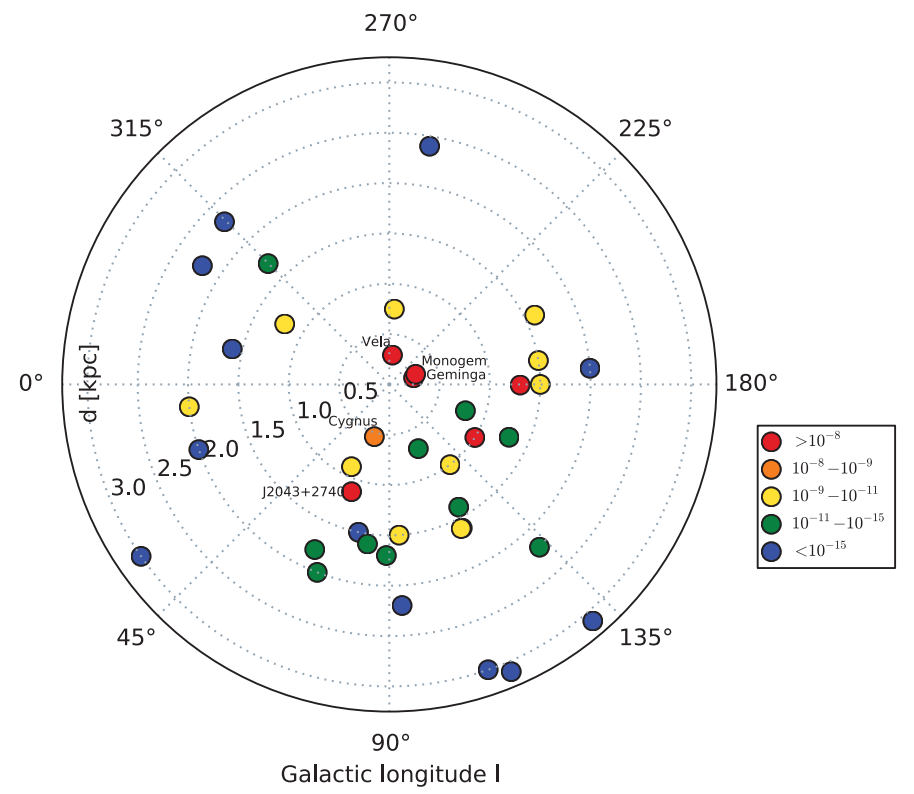

Figure 5. Sample of single SNRs and PSRs in terms of their Galactic longitude $l$ [deg] and distance to the Earth [kpc] (located at the center of the circle). The color scale of the dots quantifies the electron flux integrated from $50 \mathrm{GeV}$ to $5 \mathrm{TeV}$, in units of $\left(\mathrm{cm}^{2} \mathrm{~s} \mathrm{sr}\right)^{-1}$.
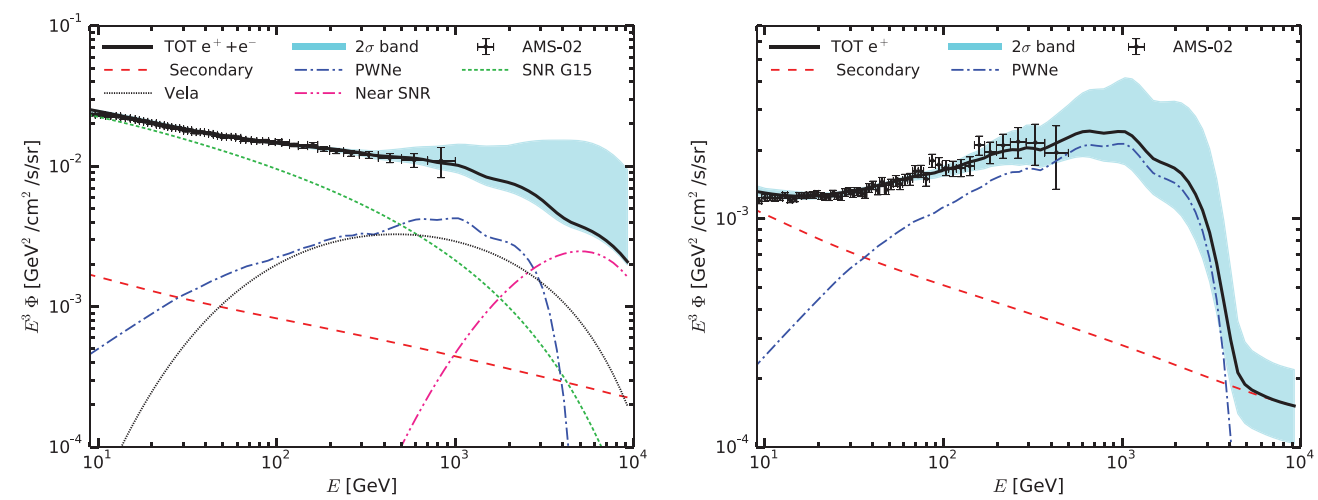

Figure 6. Fit to $e^{+}+e^{-}$(left panel) and $e^{+}$(right panel) AMS-02 data with MAX propagation model and $R_{\text {cut }}=0.7 \mathrm{kpc}$ for Case 2 (see Manconi et al. 2017 for details). All the components for the best fit are displayed together with the $2 \sigma$ uncertainty band on the total flux. Line coding as follows: solid black, sum of all the components in the plot; red dashed, secondary $e^{+}$and $e^{-}$; blue dash-dotted: $e^{+}$and $e^{-}$from all ATNF PWNe; green dotted: $e^{-}$from far SNR; black dotted: $e^{-}$from Vela SNR; magenta double dash-dotted: $e^{-}$from all other near $(R \leqslant 0.7 \mathrm{kpc})$ SNRs. The left (right) panel shows the $e^{+}+e^{-}\left(e^{+}\right)$flux.

The results for the $e^{+}+e^{-}$anisotropy are summarized in Fig. 7 as a function of $E_{\min }$ energy, and compared to Fermi-LAT upper limits. We plot $\Delta_{e^{+}} e^{-}$for the best fit on the AMS-02 data, for the Vela and Cygnus Loop SNRs. We also plot the anisotropy for Vela with its $2-\sigma$ uncertainty band. The Vela anisotropy is an increasing function of $E_{\text {min }}$, at least up to few hundreds $\mathrm{GeV}$, and depending on the model parameters. Its is predicted with an uncertainty of one order of magnitude or larger, and only mildly dependent on the bin of integrated energy. For $E_{\text {min }}$ below $200 \mathrm{GeV}$, the Fermi-LAT upper limits lie in the Vela anisotropy band, while for higher energies the experimental data they are at 


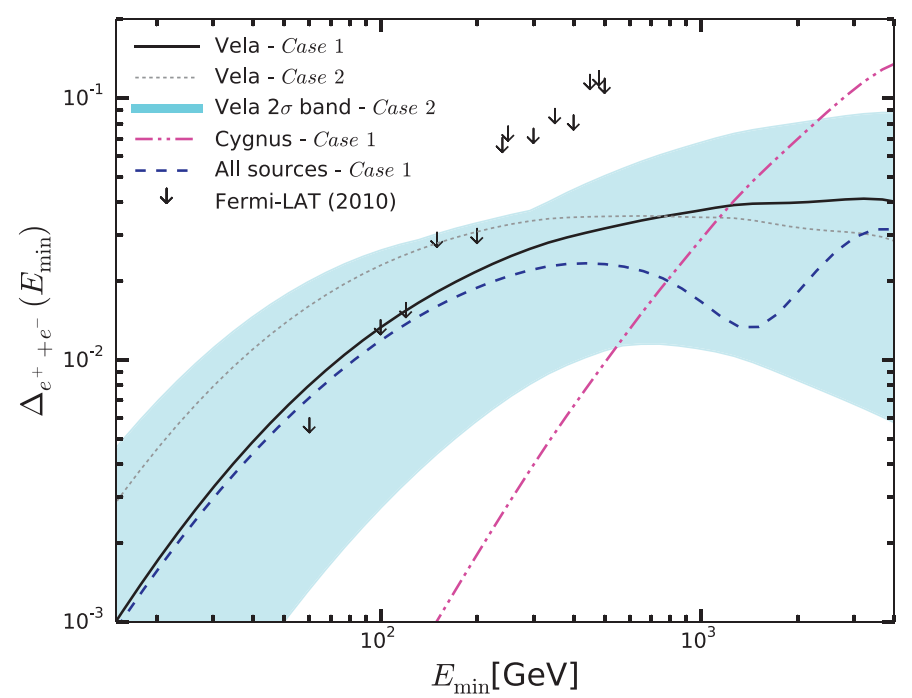

Figure 7. Predictions for the dipole anisotropy in the $e^{+}+e^{-}$flux from single SNRs, for $R_{\text {cut }}=0.7 \mathrm{kpc}$ and MAX propagation parameters. The energy bins are integrated in energy from $E_{\mathrm{min}}$ up to $5 \mathrm{TeV}$. The anisotropy from Vela and from Cygnus Loop resulting from the best fit of Case 1 analysis (see Manconi et al. 2017) are plotted as solid black and dot-dashed magenta lines, respectively. The results for the Vela best fit and 2- $\sigma$ uncertainty band within Case 2 are shown by the black dotted line and cyan band. The blue dashed curve corresponds to the anisotropy from all the single sources considered in Case 1. The downward arrows reproduce the Fermi-LAT upper limits.

least a factor of two higher than the maximal expected $\Delta_{e^{+} e^{-}}$. The Fermi-LAT upper limits have already the power to test some of the models for Vela emitting $e^{-}$compatible with AMS-02 $e^{+}+e^{-}$and $e^{+}$data.

\section{Conclusions}

The astrophysics of experimental CRs has entered an era of precision The future of astroparticle physics is bright, and can help the understanding of our Galaxy, both in its known sources and in the halo DM component. Not only the experiments become more sensitive and sophisticated, also the connections between them grow rapidly stronger. No analysis is nowadays complete without the study of counterparts in other particle types and wavelengths. The experimental search for dipole anisotropies in the lepton sector encourages the growth of an astronomy of charged CRs. The forthcoming measurements will be crucial to ascertain the presence of DM particles in the halo of our Galaxy and to hint at their particle physics nature.

\section{References}

Aramaki, T. et al. 2016, Phys. Rep. 618, 1

Castellina, A. \& Donato, F. 2012, "Planets, Stars and Stellar Systems", Springer 5, 4760, arxiv: 1110.2981

Donato, F., Fornengo, N., \& Salati, P. 2000, PRD, 62, 043003

Donato, F. et al. 2001, ApJ, 563, 172

Donato, F. et al. 2004, PRD, 69, 063501

Donato, F., Fornengo, N., \& Maurin, D. 2008, PRD, 78, 043506

Donato, F., Korsmeier, M., \& Di Mauro, M. 2017, subm. PRD, arxiv:1704.03663 
Evoli, C., Gaggero, D., Grasso, D., \& Maccione, L. 2008, JCAP 10, 18

Evoli, C. et al. 2017, JCAP, 02, 015

Green, D. A. 2015, MNRAS, 454, 1517

Kissmann, R. 2014, Astropart. Phys., 55, 37

Manconi, S., Donato, F., \& Di Mauro, M., 2017, JCAP 01, 006

Maurin, D., Donato, F., Taillet, R., \& Salati, P. 2001, ApJ 555, 585

Maurin, D. \& others 2002, astro-ph/0212111

Salati, P., Donato, F., \& Fornengo, N. 2010, Indirect dark matter detection with cosmic antimatter. Cambridge University Press 26, 521

Strong, A. W. \& Moskalenko, I. V. 1998, ApJ 509, 212

Strong, A. W., Moskalenko, I. V., \& Ptuskin, V. S. 2007, Ann. Rev. Nucl. Part. Sci. 57, 285 